\title{
Microscopic Non-Universality versus Macroscopic Universality in Algorithms for Critical Dynamics
}

\author{
U. Ritschel* and P. Czerner ${ }^{\dagger}$ \\ Fachbereich Physik, Universität GH Essen, 45117 Essen (F R Germany)
}

\begin{abstract}
We study relaxation processes in spin systems near criticality after a quench from a high-temperature initial state. Special attention is paid to the stage where universal behavior, with increasing order parameter $m(t) \sim t^{\theta}$, emerges from an early non-universal period. We compare various algorithms, lattice types, and updating schemes and find in each case the same universal behavior at macroscopic times, despite of surprising differences during the early nonuniversal stages.
\end{abstract}

PACS: 64.60.Ht,05.50.+q,02.70.Lq

*e-mail: uwe@theo-phys.uni-essen.de

†e-mail: peterc@theo-phys.uni-essen.de 
Temperature quenches in spin systems and the ensuing relaxational processes have been a much-studied subject in the recent past [1 19]. Especially for a quench from a hightemperature initial state to the critical region, a new universal regime was predicted by Janssen et al. [2]. In its most pronounced form this phenomenon, termed universal shorttime behavior (USTB) in the following, occurs in a model with purely relaxational dynamics (model A according to Ref. [20]). Starting from an initial state with $T \gg T_{c}$ and a small initial magnetization $m_{0}$, the magnetization increases as [2]

$$
m(t) \sim m_{0} t^{\theta}
$$

for a macroscopic time span before it reaches a maximum and eventually decays to the equilibrium value zero. $\theta$ is an independent new exponent determined by the non-equilibrium initial state that cannot be expressed in terms of equilibrium exponents. Its value in the two-dimensional Ising system for instance is $\theta \simeq 0.19$ [6, 15].

The results of Janssen et al. [2,3] allowed the interpretation of earlier Monte Carlo (MC) simulations [4], and later the power law (11) was also directly verified in the "computer experiment" [5]. Further it was pointed out by Li et al. [6] that the USTB can be exploited to determine equilibrium exponents from the early non-equilibrium. In the sequel, this method was further developed and applied to a number of systems [7,8]. Moreover, USTB in other dynamic universality classes [9], in systems with a tricritical point [10], and in disordered and dilute spin systems was studied [11]. Finite size effects were analyzed in Refs. [12,13], and the USTB near surfaces was studied in Ref. [14]. Further, the close relationship between USTB and "damage spreading" was pointed out [15], USTB in a different context, for quasilong-range order evolving after a quench to the Kosterlitz-Thouless phase, was investigated [16], and a general scaling invariance in the short-time regime was found [17]. Possibly also related to USTB is the "overshooting" of the order parameter beyond the equilibrium domain magnetization for quenches below $T_{c}$ [18], and the issue whether there exist still more independent exponents for relaxational processes was raised in a recent preprint 19.

A simple physical argument for the growth of the magnetization in (1) was given by Janssen [3,21]. Consider a system that is quenched to some final temperature $T_{f}$ (not necessarily $T_{c}$ ), again with initial magnetization $m_{0}$. Then for $T_{f} \ll T_{c}, m(t)$ should grow after the quench, towards the equilibrium value selected by $m_{0}$. If in contrast $T_{f} \gg T_{c}, m(t)$ is expected to decay to zero rapidly. Hence, there should be a limiting temperature $T_{l}$ where the qualitative behavior changes.

As the initial correlations are short-ranged, the natural candidate for $T_{l}$ is the critical temperature of the mean-field (MF) theory $T_{c}^{M F}$, and with the real $T_{c}$ of spin systems being always smaller than $T_{c}^{M F}$, it would be an immediate consequence that $m(t)$ increases for a quench to the critical point. However, as argued in Ref. [13], it is not possible to derive the power law (1) from this scenario. The power-law growth is rather a phenomenon that occurs when the time-dependent (growing) correlation length $\xi(t)$ has become macroscopic, i.e., much larger than the lattice spacing a (compare Fig. 1 below). The derivation of (1) is thus beyond the scope of MF theory.

So far numerical investigations have been mostly carried out with the heat-bath (HB) algorithm 22,23]. A comparison between $\mathrm{HB}$ and Metropolis (ME) algorithm [22] was performed for the Potts model by Okano et al. [8], and it turned out that concerning the universal behavior both algorithms yield compatible results, but differences occur for early 
times.

The main purpose of this Letter is a more systematic examination of the issue of universality. Are equilibrium exponents determined with the USTB really independent of factors like the algorithm (HB or ME), the updating scheme (random or sequential), and the lattice type (nearest or next-nearest neighbor coupling, square or triangular lattice)? How does universal behavior in the regime with $\xi(t) \gg a$ emerge from the non-universal early stage with $\xi(t) \simeq a$ ? And closely related: Is it really a mean-field ordering process during the microscopically early stages, or has this simple picture to be refined?

We answered these questions by solving the master equation for early times (during the first single-spin updates) as well as by MC simulation for later times. Our work reveals a number of interesting and surprising details about algorithms for critical dynamics and puts the USTB as a method to access equilibrium properties from a non-equilibrium (though universal) regime on a much firmer basis.

Information about thermal averages in the early non-universal stage of the relaxational process can be obtained by solving the master equation [22]

$$
\frac{d P(\mathbf{s}, t)}{d t}=\sum_{\mathbf{s}^{\prime}}\left[W\left(\mathbf{s}^{\prime} \rightarrow \mathbf{s}\right) P\left(\mathbf{s}^{\prime}, t\right)-W\left(\mathbf{s} \rightarrow \mathbf{s}^{\prime}\right) P(\mathbf{s}, t)\right]
$$

where $\mathbf{s}$ denotes a spin configuration, $W\left(\mathbf{s}^{\prime} \rightarrow \mathbf{s}\right)$ is the transition probability, $P(\mathbf{s}, t)$ the probability to find configuration $\mathbf{s}$ at time $t$, and the sum extends over all possible configurations. The analytic integration of Eq. (2) for large systems of coupled spins is not feasible. However, for the high-temperature initial state consisting of $N$ uncorrelated spins in a magnetic field $H$, characterized by the Boltzmann factor

$$
P(\mathbf{s}, 0)=Z_{0}^{-1} \exp \left(H \sum_{i=1}^{N} s_{i}\right) \quad \text { with } \quad Z_{0}=(2 \cosh H)^{N},
$$

the analytic treatment for very early times, $t \ll 1$, is possible. (The time is expressed in units of MC steps per site (MCS).)

Consider the first single-spin update after the quench. It takes place in an environment that is coupled to a heat bath at the final temperature $T_{f}$, and $H$ is switched off. Decisive for the very early stage is whether after the first update on average the magnetization is reduced or increased. The respective tendency survives as long as the system still closely resembles the initial state, i.e., as long as the number of single-spin updates is much smaller than $N$.

Without loss of generality one may choose spin 1 to be updated. From (2) one straightforwardly derives $\Delta m:=m(t=1 / N)-m_{0}$ to be

$$
\Delta m=2 N^{-1} \sum_{\mathbf{s}}[W(-\rightarrow+) P(-, 0)-W(+\rightarrow-) P(+, 0)]
$$

where the $W$ 's are the probabilities for spin 1 changing sign with all other spins remaining unchanged. Especially for small $H$ (corresponding to small $m_{0}$ ), we find from (雨) the simple result

$$
\Delta m=-4 H N^{-1} \sum_{\mathbf{s}} W(+\rightarrow-)\left(1+\sum_{i \in \mathrm{IN}} s_{i}\right)
$$


where the second sum extends over the interacting neighbors of spin 1.

From (5) we calculated $\Delta m$ and the limiting temperature $T_{l}$ for the Ising model on a square lattice with nearest-neighbor interaction $J$ for HB and ME algorithm. In $d=2$ (the system that will be also studied by MC simulations below) the explicit results are $T_{l}^{H B}=3.0898 \ldots$ and $T_{l}^{M E}=1.5885 \ldots$ for $\mathrm{HB}$ and ME algorithm, respectively. (Temperatures are expressed in units of $J / k_{B}$.) For comparison, the (exact) critical temperature is $T_{c}=2.2691 \ldots$ and $T_{c}^{M F}=4$. Hence, for the HB algorithm $T_{l}$ is indeed above $T_{c}$, while, surprisingly, with $\mathrm{ME}$ even at $T_{c}$ the magnetization decays $t \ll 1$.

We calculated $T_{l}$ also for other dimensionalities. In the limit $d \rightarrow \infty$ (where the number of nearest neighbors becomes infinite) one finds $T_{l} \rightarrow T_{c}^{M F}$ for both algorithms considered above. For $d=1$ both yield $T_{l}=0$.

From this analysis we conclude: First, the limiting temperature in general depends on the algorithm. Only for $d \rightarrow \infty$ it turns out to be $T_{c}^{M F}$. Second, we are left with the puzzling result that for the ME algorithm the limiting temperature lies even below $T_{c}$, and therefore one would not expect to see an increase of the magnetization at $T_{c}$. In any event, the simple explanation that the non-universal stage preceding the USTB is a MF ordering process is in general not correct.

In order to learn about later stages, $t \gtrsim 1$, especially the crossover from microscopic to macroscopic behavior, we had to resort to $\mathrm{MC}$ simulations. These were carried out for an Ising system on a square lattice with linear dimension $L$ and periodically coupled boundaries. Single spins were randomly selected and updated. In order to obtain thermal expectation values we generated a large number of histories, each starting from a new initial configuration, and calculated mean values [22].

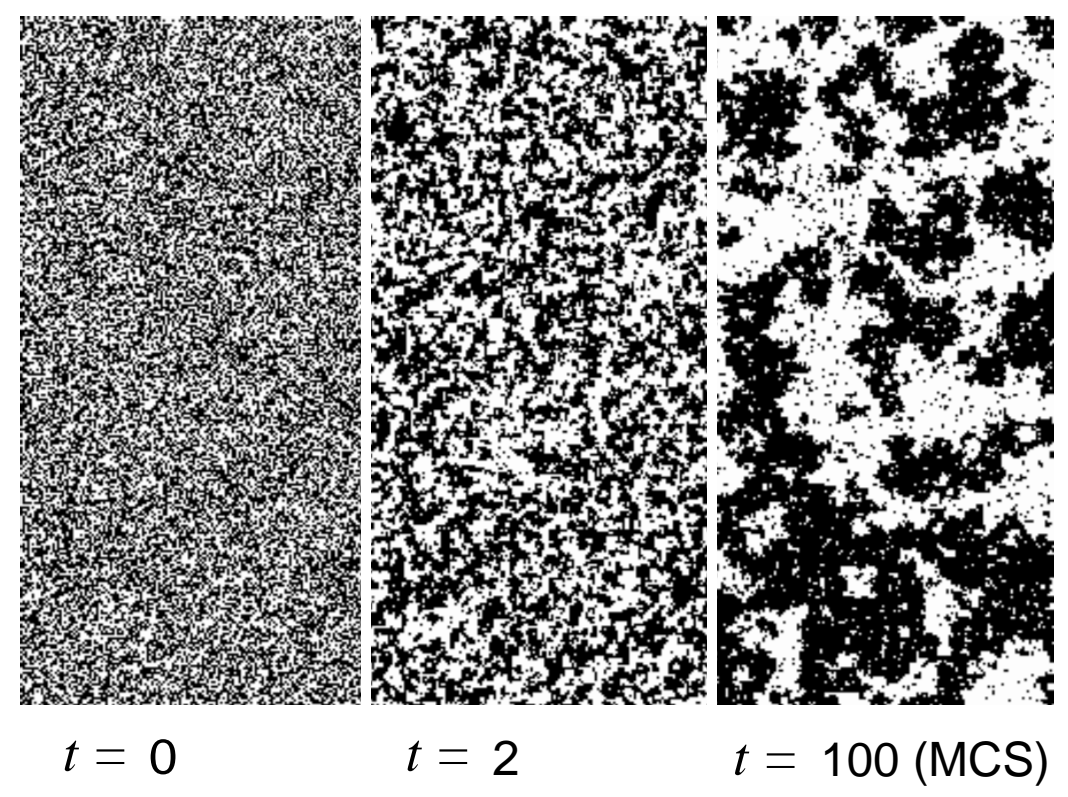

Fig. 1: Three snapshots of the temporal evolution of a spin configuration for $L=300$ and $m_{0}=0$, generated with the HB algorithm and random updating. Displayed in each picture is half of the system. From the visual appearance there is no difference HB and ME algorithm. 
Snapshots of the temporal evolution of a single configuration for a square lattice with $N=90000$ spins are displayed in Fig. 1. The left picture shows the initial state. Next to it the configuration after $2 N$ updates corresponding to $t=2$ is depicted. At this point the average domain size and (with that) the correlation length are already substantially larger than the lattice spacing. This is the stage where universal (macroscopic) behavior emerges from the non-universal (microscopic) regime as discussed in more detail below. At $t=100$ the correlation length is of the order of the lattice size $L$.

Results for $m(t)$ at $T_{f}=T_{c}$ with $L=20$ and $m_{0}=0.05$ are displayed in Fig. 2 . The HB curve (solid line) monotonously increases and is consistent with a power-law for $t \gtrsim 1.5$. In the case of the ME algorithm (dashed line) the behavior is qualitatively different. As expected from the our analytic results, $m(t)$ indeed drops initially, but has a minimum at $t \simeq 0.3$, and then increases to assume the power-law form for $t \gtrsim 2$. Thus, despite of the anomalous time dependence of the ME curve in the non-universal regime, for macroscopic times it agrees with (1). This is in accord with the findings of Okano et al., where sequential updating was used and, thus, the details of the temporal evolution were not uncovered. Later the profiles in Fig. 2 have a maximum and then decay to the equilibrium value zero 24.

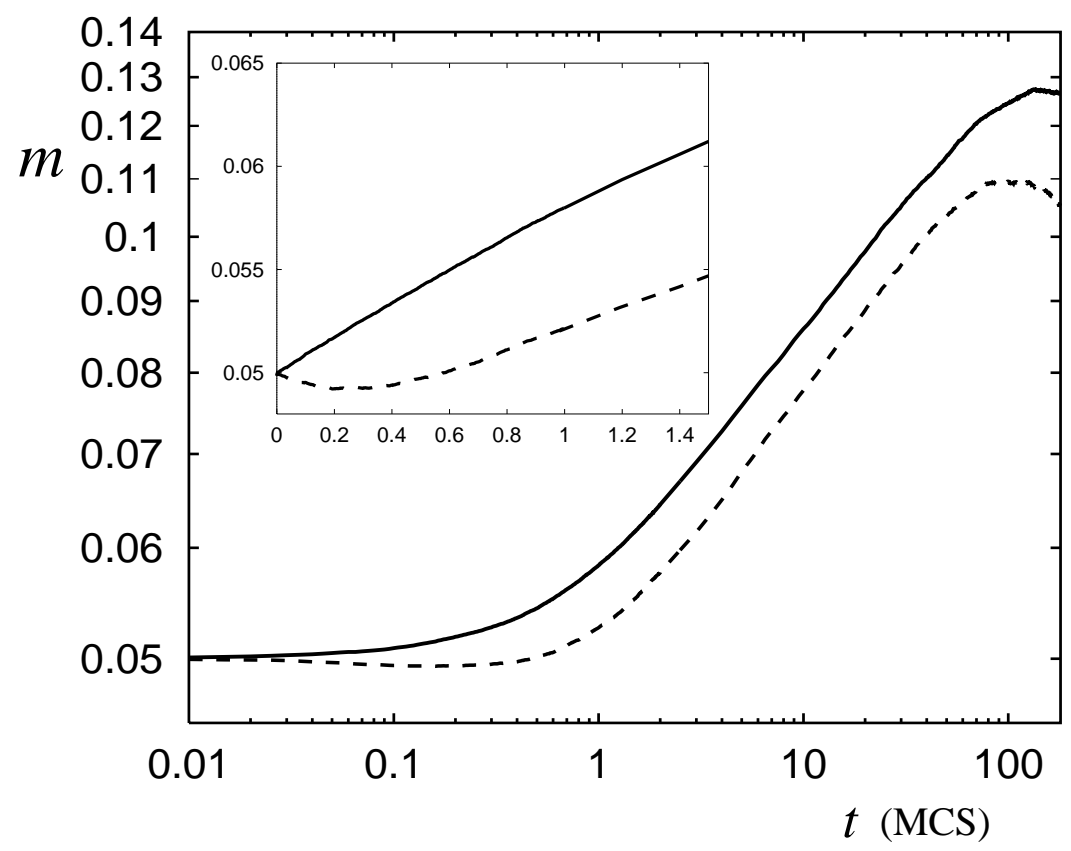

Fig. 2: Order-parameter profiles for $L=20$ and $m_{0}=0.05$ obtained with HB (solid line) and ME algorithm (dashed line) in double-logarithmic representation. The small diagram inserted shows the data for small times in double-linear representation.

Taking into account these results, the natural question to ask is whether there exists an algorithm-independent limiting temperature, a "dynamic MF temperature", where the macroscopic behavior changes from increasing (USTB) to decaying. It turns out that for the HB algorithm this limiting value coincides with $T_{l}^{H B}$; the profiles have mostly one extremum. In order to determine the corresponding limit for $\mathrm{ME}$, we generated a number of profiles for temperatures above $T_{c}$, seeking the one that shows a saddle point. We detemined the 
corresponding temperature as 2.70 (2). This number did neither depend significantly on the system size nor on $m_{0}$. However, it does not agree with the corresponding value of the HB dynamics $T_{l}^{H B} \simeq 3.1$.

Eventually we compared magnetization profiles in the universal regime for a system with $L=40$ and $m_{0}=0.03$ at the critical point, $T_{f}=T_{c}$, for various combinations of algorithms, lattice types, and updating schemes. For the square lattice with nearest-neighbor interactions we combined ME and $\mathrm{HB}$ algorithm with random and sequential updating (4 curves), for the triangular lattice with nearest-neighbor interactions we used both algorithms and sequential updating (2 curves), and for a square lattice with additional next-nearestneighbor interactions we used the HB algorithm and sequential updating (1 curve) [25].

As can be seen already from Fig. 2, even though the initial power law is assumed by both profiles depicted there, the heights and locations of the maxima depend on the details of the method, besides the differences for early times. However, in all cases studied it turned out to be possible to map the data onto a single curve for times $t \gtrsim 20$, by constant rescalings both axes. The result is shown in Fig. 3. On the semi-logarithmic plot (small insertion) the individual profiles cannot be distinguished. When both axes are plotted logarithmically, on the other hand, the short-time regime is more pronounced, and significant differences for $t \lesssim 10$ become visible. The pure power law (solid line above the data) is plotted for comparison.

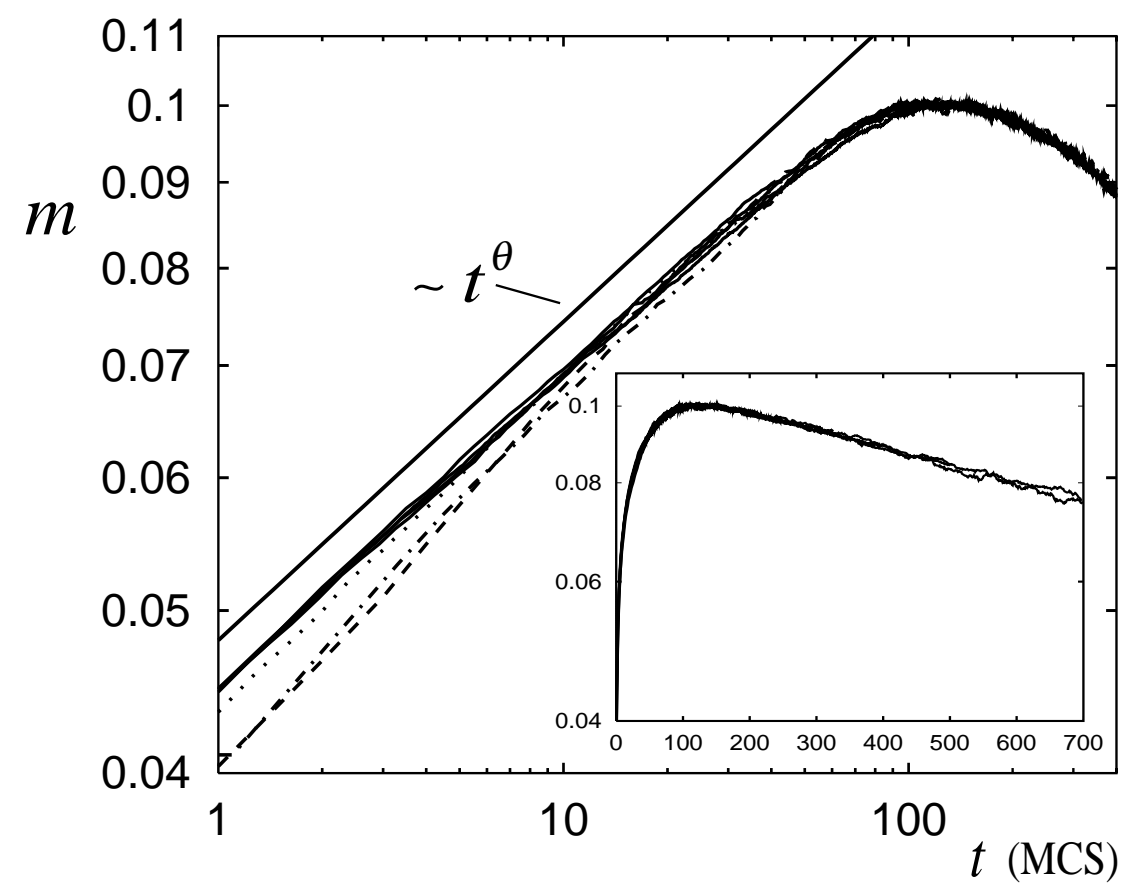

Fig. 3: Data collapse of seven magnetization profiles obtained for $L=40$ and $m_{0}=0.03$ for different combinations of lattice types, algorithms, and updating schemes (as described in the text) in double-logarithmic representation. The ME results for the square lattice with sequential (dashed line) and random (dotted line), as well as for the triangular lattice with sequential update (dasheddotted line) are singled out. The small diagram inserted shows the same data in semi-logarithmic representation. The power law (11) with $\theta=0.19$ is plotted for comparison. 
Singled out are the ME curves, for the square lattice with sequential (dashed) and random (dotted) updating, and for the triangular lattice with sequential updating (dashed-dotted). In particular when the ME algorithm is combined with sequential updating, the power law is assumed only for $t \gtrsim 10$. This is consistent with the findings of Okano et al. [8] for the Potts model, and can now be interpreted as a consequence of the anomalous behavior of the $\mathrm{ME}$ algorithm for $t \lesssim 1$. The HB results assume the power-law form much earlier. Most importantly, however, all seven curves show the USTB as expressed in (四) for later times, $t \gtrsim 10$.

In conclusion, we studied the short-time behavior in relaxational processes after a temperature quench. We found surprisingly different temporal evolutions with heat-bath and Metropolis algorithm during early, non-universal stages. Nevertheless, the characteristic short-time power law (1) turned out to be a rather robust phenomenon, occurring independently of the algorithm, the lattice type, and the updating scheme, provided the systems belong to the same dynamic universality class - the Ising system with short-range interactions and non-conserved order parameter was the example studied above - and the correlation length has grown substantially larger than microscopic scales.

Acknowledgements: This work was supported in part by the Deutsche Forschungsgemeinschaft through Sonderforschungsbereich 237 "Unordnung und große Fluktuationen". 


\section{REFERENCES}

[1] See A. J. Bray, Advances in Physics, 43, 357 (1994) for a recent review on phase-ordering kinetics after quenches to low temperatures.

[2] H. K. Janssen, B. Schaub, and B. Schmittmann, Z. Phys. B 73, 539 (1989).

[3] H. K. Janssen, in From Phase Transitions to Chaos - Topics in Modern Statistical Physics, edited by G. Györgyi, I. Kondor, L. Sasvári, and T. Tel (World Scientific, Singapore, 1992).

[4] D. A. Huse, Phys. Rev. B 40, 304 (1989); see also K. Humayun and A. J. Bray, J. Phys. A: Math. Gen. 24, 1915 (1991).

[5] Z.-B. Li, U. Ritschel and B. Zheng, J. Phys. A: Math. Gen. 27, L837 (1994).

[6] Z.-B. Li, L. Schülke, B. Zheng, Phys. Rev. Lett. 74, 3396 (1995); Phys. Rev. E 53, 2940 (1996)

[7] L. Schülke and B. Zheng, Phys. Lett. A 204, 295 (1995), ibid. A 215, 81 (1996).

[8] K. Okano, L. Schülke, K. Yamagishi, and B. Zheng, Universality and Scaling in ShortTime Dynamics, Siegen preprint (1995).

[9] K. Oerding and H. K. Janssen, J. Phys. A: Math. Gen. 26, 3369 (1993); ibid. 26, 5295 (1993).

[10] H. K. Janssen and K. Oerding, J. Phys. A: Math. Gen. 27, 715 (1994).

[11] K. Oerding, J. Stat. Phys. 78, 893 (1995); J. Phys. A: Math. Gen. 28, L639 (1995); K. Oerding and H. K. Janssen, J. Phys. A 28, 4271 (1995); H. K. Janssen, K. Oerding, and E. Sengespeick, J. Phys. A 28, 6073 (1995).

[12] H. W. Diehl and U. Ritschel, J. Stat. Phys. 73, 1 (1993); U. Ritschel and H. W. Diehl, Phys. Rev. E 51, 5392 (1995).

[13] U. Ritschel and H. W. Diehl, Nucl. Phys. B 464, 512 (1996).

[14] U. Ritschel and P. Czerner, Phys. Rev. Lett. 75, 3882 (1995); S. N. Majumdar and A. M. Sengupta, Phys. Rev. Lett. 76, (1996).

[15] P. Grassberger, Physica A 214, 547 (1995).

[16] P. Czerner and U. Ritschel, Phys. Rev. E 53, 3333 (1996).

[17] B. Zheng, Phys. Rev. Lett. 77, 679 (1996).

[18] H. Gilhøj, C. Jeppesen, and O. G. Mouritsen, Phys. Rev. Lett 75, 3305 (1995).

[19] S. N. Majumdar, A. J. Bray, S. J. Cornell, and C. Sire, Global persistence exponent for critical dynamics, cond-mat/9606123.

[20] P. C. Hohenberg and B. I. Halperin, Rev. Mod. Phys. 49, 435 (1977).

[21] H. K. Janssen, private communication.

[22] K. Binder and D. W. Heermann, Monte Carlo Simulation in Statistical Physics, (Springer, Berlin, 1988) provides a good introduction in the dynamic interpretation of Monte Carlo simulations and the different algorithms used.

[23] For descriptions of the heat-bath algorithm see B. Derrida and G. Weisbuch, Europhys. Lett. 4, 657 (1987) and A. M. Mariz, H. J. Herrmann, and L. de Arcangelis, J. Stat. Phys. 59, 1043 (1990). For the MC simulations reported in this work the heat-bath algorithm is equivalent to the original Glauber dynamics, see A. M. Mariz and H. J. Herrmann, J. Phys. A 22, L1081 (1989).

[24] For a detailed discussion of the later stages, especially the time scales which determine the location of the maximum and the rate of decay to the equilibrium, we refer to Refs. [5, 12]. 
[25] For the triangular lattice the exact critical temperature is $T_{c}=3.6409 \ldots$ For the square lattice with additional next-nearest-neighbor interactions we chose (for simplicity) the additional coupling to be equal to $J$ (the nearest-neighbor coupling). We determined the critical temperature for this system as $T_{c} \simeq 5.26$. 